Оригинальная статья/Original article

УДК 360

DOI: http://doi.org/10.20914/2310-1202-2017-2-337-345

Формирование потенциала научно-производственного комплекса

\begin{tabular}{|c|}
\hline $\begin{array}{lll}\text { Елена А. Федорова } & 1 & \text { eafokt@rambler.ru } \\
\text { Андрей Ю. Горбачев } & 2 & \text { merc123@mail.ru }\end{array}$ \\
\hline $\begin{array}{l}\text { ООО «Универсал-Аудит», ул. Оружейная, 5а, г. Тула, 300012, Россия } \\
\text { АО Центральное конструкторское бюро аппаратостроения, ул. Демонстрации, 36, г. Тула, } 3\end{array}$ \\
\hline 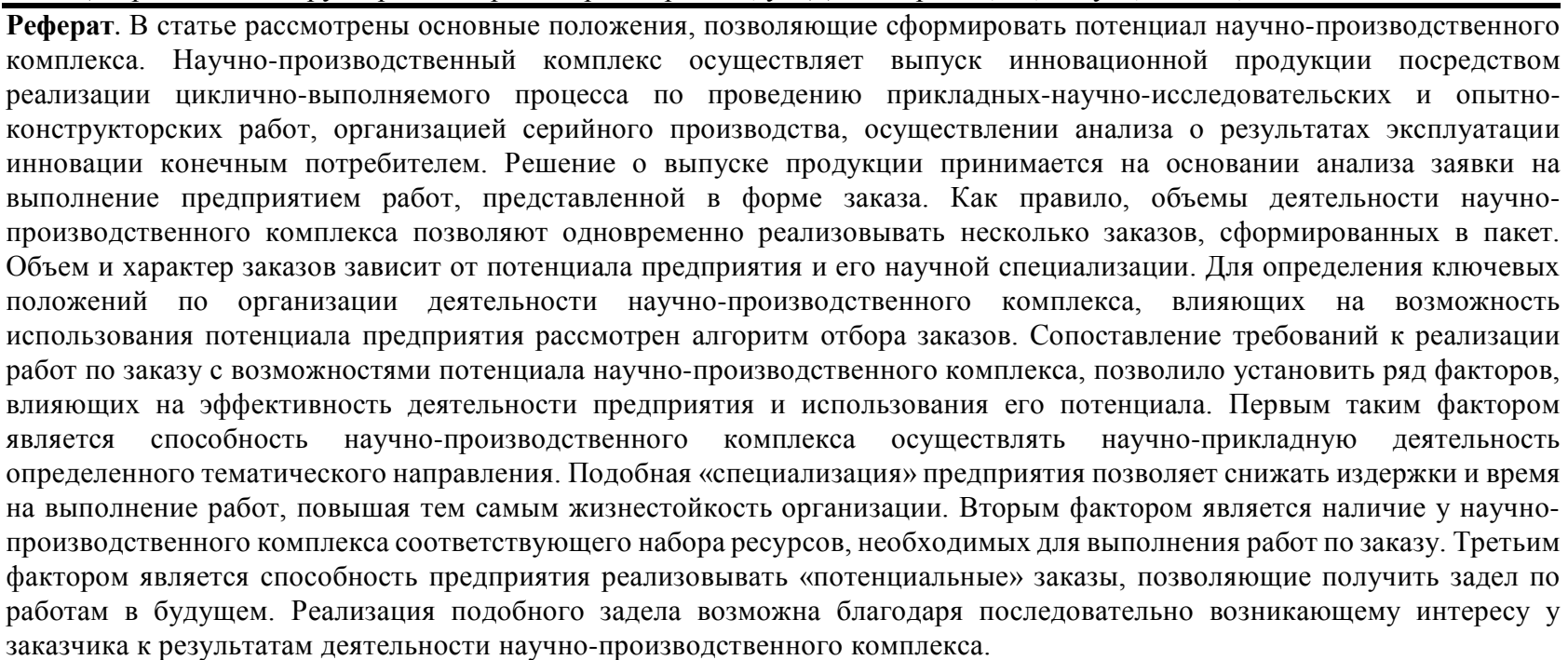 \\
\hline
\end{tabular}

\title{
Formation of potential of the scientific-industrial complex
}

\begin{tabular}{lll}
\hline \hline Elena A. Fedorova & 1 & eafokt@rambler.ru \\
& Andrey Yu. Gorbachev ${ }^{2}$ & merc123@mail.ru \\
\hline
\end{tabular}

$\overline{1}$ “Universal-Audit”, Oruzheynaya St., 5a, Tula, 300012, Russia

${ }^{2}$ The Central design office machine construction, Demonstration str., 36, Tula, 300034, Russia

Summary. In article the basic provisions allowing to create the capacity of scientific-industrial complex are considered. The scientific-industrial complex makes release of innovative production by means of realization of the cyclic carried-out process on carrying out applied - research and development, the organization of mass production., implementation of the analysis about results of operation of an innovation by the end user. The decision on production is made on the basis of the analysis of the request for performance of works by the enterprise submitted in the form of the bespoke. As a rule, volumes of activity of scientific-industrial complex allow to realize at the same time several bespoke created in a package. The volume and character of orders depends on the capacity of the enterprise and its scientific specialization. For definition of the key provisions on the organization of activity of scientific-industrial complex influencing a possibility of use of capacity of the enterprise the algorithm of selection of bespoke is considered. Comparison of requirements to realization of works on the order to opportunities of capacity of scientific-industrial complex, allowed to establish a number of the factors influencing efficiency of activity of the enterprise and use of its potential. The first such factor is ability of scientific-industrial complex to carry out scientific and applied activity of a certain thematic direction. Similar "specialization" of the enterprise allows to reduce costs and time for performance of work, increasing thereby resilience of the organization. The second factor is presence at scientific-industrial complex of the corresponding set of the resources necessary for performance of work by bespoke. The third factor is ability of the enterprise to realize the "potential" bespoke allowing to receive a reserve on works in the future. Realization of a similar reserve is possible thanks to consistently arising interest at the customer in results of activity of scientific-industrial complex.

Keywords: potential of scientific-industrial complex, bespoke, innovation

Для цитирования

Федорова Е.А., Фамилия И.О. Формирование потенциала научнопроизводственного комплекса // Вестник ВГУИТ. 2017. Т. 79. № 2 C. 337-345. doi:10.20914/2310-1202-2017-2-337-345
For citation

Fedorova E.A., Gorbachev A.Yu. Formation of potential of the scientific-industrial complex. Vestnik VGUIT [Proceedings of VSUET]. 2017. vol. 79. no. 2. pp. 337-345. (in Russian). doi:10.20914/23101202-2017-2-337-345 


\section{Введение}

Выпуск инновационной продукции требует проведения исследовательских, конструкторских и производственных работ.

Одной из наиболее эффективных структур, позволяющих осуществлять выпуск инновационной продукции, является научно-производственный комплекс (далее НПК), представляющий собой оптимальное сосредоточение ресурсов по осуществлению следующих работ:

- прикладные научно-исследовательские (далее НИР), позволяющие получить теоретическое решение;

- опытно-конструкторские (далее ОКР), результат которых состоит в изготовлении опытного образца на основании данных НИР;

- серийное производство, позволяющее осуществлять массовый выпуск продукции;

- анализ данных о результатах эксплуатации инновации конечным потребителем, производимый с целью определения необходимости, способов и возможностей дальнейшего развития проекта, совершенствовании и модернизации осуществленных разработок.

Инновационная деятельность НПК циклический и постоянно развивающийся процесс, обусловленный факторами научно-технического прогресса и конкурентной борьбой между производителями за рынок.

Рост финансирования государственных затрат и средств бизнеса на исследования и разработки инновационной продукции, согласно данным российского статистического ежегодника за 2016 г. (таблицы 1 и 2) в РФ существует высокая потребность в научных исследованиях.

Как следствие, рассмотрение эффективных способов организации деятельности НПК, позволяющей реализовывать выпуск инновационный выпуск продукции, является актуальной задачей.

Объемы деятельности НПК позволяют одновременно реализовывать несколько заказов, сформированных в пакет. Объем и характер пакета заказов зависит от потенциала предприятия и его научной специализации.

Потенциал НПК определяется его ресурсами, которые имеют свою структуру образования и используются в процессе производства инновационной продукции.

К ресурсам НПК можно отнести:

1. Технологические процессы - технологические и технические решения проведения научно-исследовательских и производственных работ. Логическая цепочка технологических процессов, позволяет реализовать проект по производству инновационной продукции $[1,9]$.

Технологические процессы могут быть реализованы НПК ранее в результате предыдущей деятельности. Тогда, при разработке новой технологической цепочки для вновь поступившего заказа, ранее реализованные технологические процессы, применяемые в новом проекте, считаются активом предприятия, позволяющего снизить издержки на разработку и выпуск инновационного товара.

2. Кадровый ресурс - персонал, соответствующей численности и квалификации участвующий, в проведении работ.

Ввиду того, что инновационная детальность НПК связана с двумя крупными видами выполняемых операций - прикладные научноисследовательские и производственные работы персонал предприятия также может быть соответствующе классифицирован:

- исследовательская группа, осуществляющая разработку и внедрение технологических процессов, необходимых для выполнения заказа;

- производственная группа, осуществляющая производственные операции.

3. Материально-техническая база - набор оборудования, включая также вспомогательные площади, необходимые для его размещения, позволяющего производить требуемый тип работ, а именно:

- исследовательское оборудование, позволяющее осуществлять научно-прикладные исследования;

- производственное оборудование, включающее станки, приспособления и прочее, позволяющее осуществлять производственный процесс.

4. Финансовые ресурсы - объем средств которыми располагает НПК для проведения работ.

Совокупность ресурсов НПК образует его потенциал - способность реализовывать максимально возможный объем заказов, выраженный в стоимостном выражении, за определенный период, посредством наилучшего использования имеющихся ресурсов. 
Затраты государства (РФ) на исследования и разработки (миллионов рублей)

Costs of the state of (RF) researches and developments (millions of rubles)

\begin{tabular}{|c|c|c|c|c|c|c|}
\hline & 2010 & 2011 & 2012 & 2013 & 2014 & 2015 \\
\hline $\begin{array}{l}\text { 1. Внутренние затраты на исследования и разработки } \\
\text { 2. Domestic expenditures on research and development }\end{array}$ & 523377 & 610427 & 699870 & 749798 & 847527 & 914669 \\
\hline \multicolumn{7}{|l|}{ в том числе: including: } \\
\hline \multicolumn{7}{|l|}{ 1.1. По социально-экономическим целям: Socio-economic objectives: } \\
\hline - развитие экономики development of the economy & 183114 & 231942 & 295902 & 303849 & 319943 & 335508 \\
\hline $\begin{array}{l}\text { - сельское хозяйство, лесоводство, рыболовство } \\
\text { agriculture, forestry, fishing }\end{array}$ & 12090,8 & 15011,6 & 16151,6 & 18162,6 & 19356,1 & 20507,9 \\
\hline $\begin{array}{l}\text { - производство, распределение и рациональное } \\
\text { production, distribution and rational }\end{array}$ & 19174,8 & 19913,7 & 30798,2 & 32391,1 & 30009,8 & 28233,8 \\
\hline \multicolumn{7}{|l|}{ 1.2. Использование энергии: Use of energy: } \\
\hline - промышленное производство industrial production & 126029 & 156696 & 200460 & 211581 & 225974 & 247190 \\
\hline - строительство construction & 5513,7 & 7295 & 8504,2 & 5514,1 & 7302,9 & 4100,7 \\
\hline - транспорт transport & 12686 & 22041,6 & 23986,2 & 26950,4 & 25052,8 & 28936,9 \\
\hline - связь link & 6704,7 & 9211,9 & 14147,2 & 7577,7 & 10809 & 5137 \\
\hline $\begin{array}{l}\text { 2. Инфраструктура и планировка городских и сельских населенных пунктов } \\
\text { Infrastructure and layout of urban and rural settlements }\end{array}$ & 354,5 & 917 & 912,5 & 702,1 & 754 & 720,4 \\
\hline 3. Сфера услуг Service sector & 559,8 & 855,1 & 942,3 & 969,7 & 685,1 & 681 \\
\hline 4. Социальные цели Social goals & 24966,2 & 29640,4 & 33070,8 & 39876,8 & 45328,4 & 47512,6 \\
\hline \multicolumn{7}{|l|}{ в том числе: including: } \\
\hline 4.1. Охрана окружающей среды Environmental protection & 5950 & 6638,2 & 6972,1 & 6139,3 & 7690,7 & 7698,8 \\
\hline 4.2. Охрана здоровья населения Health of the population & 14373,7 & 17100,4 & 19694,7 & 22382,8 & 27197,5 & 27779,2 \\
\hline $\begin{array}{l}\text { 4.3. Социальное развитие и общественные структуры } \\
\text { Social development and social structure }\end{array}$ & 4642,5 & 5901,8 & 6403,9 & 11354,8 & 10440,3 & 12034,6 \\
\hline 5. Общее развитие науки General development of science & 104295 & 111948 & 117873 & 130695 & 136415 & 145154 \\
\hline $\begin{array}{l}\text { 6. Исследование и использование Земли и атмосферы } \\
\text { The exploration and use of Earth and atmospheric }\end{array}$ & 19821,8 & 20390,7 & 25474,6 & 32889,9 & 39068,7 & 43206,9 \\
\hline $\begin{array}{l}\text { 7. Использование космоса в мирных целях } \\
\text { The use of outer space for peaceful purposes }\end{array}$ & 27503,7 & 35752,5 & 37559 & 51558,4 & 48996,2 & 57441,3 \\
\hline 8. Другие цели Other purposes & 163677 & 180754 & 189990 & 190928 & 257776 & 285846 \\
\hline
\end{tabular}


Таблица 2.

Внутренние затраты на исследования и разработки по источникам формирования в РФ

(миллионов рублей)

Table 2 .

Internal costs of researches and developments on formation sources in the Russian Federation

(millions of rubles)

\begin{tabular}{|c|c|c|c|c|c|c|c|}
\hline & 2000 & 2010 & 2011 & 2012 & 2013 & 2014 & 2015 \\
\hline $\begin{array}{l}\text { Внутренние затраты на исследования и разработки } \\
\text { Domestic expenditures on research and development }\end{array}$ & \begin{tabular}{|l|l|}
76 \\
697,10 \\
\end{tabular} & \begin{tabular}{|l|l|}
523 \\
377,20 \\
\end{tabular} & $\begin{array}{ll}610 \\
426,70 \\
\end{array}$ & \begin{tabular}{|l|l|}
699 \\
869,80
\end{tabular} & \begin{tabular}{|l|l|}
749 \\
797,60 \\
\end{tabular} & \begin{tabular}{|l|l|}
847 \\
527,00 \\
\end{tabular} & $\begin{array}{ll}914 \\
669,10\end{array}$ \\
\hline \multicolumn{8}{|c|}{\begin{tabular}{l|l} 
в том числе по источникам финансирования: including on & \\
financing sources: &
\end{tabular}} \\
\hline 1. Средства бюджета Budget funds & $\begin{array}{ll}41 \\
190,90 \\
\end{array}$ & $\begin{array}{l}360 \\
334,20 \\
\end{array}$ & $\begin{array}{ll}400 \\
235,70\end{array}$ & \begin{tabular}{|l|l}
462 \\
203,20
\end{tabular} & $\begin{array}{l}493 \\
470,40\end{array}$ & \begin{tabular}{|ll}
569 & 055 \\
80
\end{tabular} & \begin{tabular}{|l|l|}
617 \\
275,70
\end{tabular} \\
\hline $\begin{array}{l}\text { 2. Собственные средства научных организаций } \\
\text { Own funds of scientific organizations }\end{array}$ & 6947,20 & \begin{tabular}{|l|l|}
47 \\
407,60 \\
\end{tabular} & \begin{tabular}{|l|l|}
73 \\
293,50 \\
\end{tabular} & \begin{tabular}{|l|l|}
78 \\
520,60 \\
\end{tabular} & $\begin{array}{ll}90 \\
480,30 \\
\end{array}$ & \begin{tabular}{|l|}
99 \\
747,70 \\
\end{tabular} & $\begin{array}{l}109 \\
915,60 \\
\end{array}$ \\
\hline $\begin{array}{l}\text { 3. Средства внебюджетных фондов Funds extra-budgetary } \\
\text { funds }\end{array}$ & 4969,70 & \begin{tabular}{|l|l|}
10 \\
140,00 \\
\end{tabular} & 8808,50 & $\begin{array}{ll}11 \\
675,60 \\
\end{array}$ & $\begin{array}{ll}11 \\
777,00 \\
\end{array}$ & 9 099, 80 & 8764,20 \\
\hline $\begin{array}{l}\text { 4. Средства организаций предпринимательского сектора } \\
\text { Funds of organizations of the business sector }\end{array}$ & $\begin{array}{l}14 \\
326,20 \\
\end{array}$ & \begin{tabular}{|l|l|}
85 \\
863,30 \\
\end{tabular} & $\begin{array}{l}99 \\
408,10 \\
\end{array}$ & \begin{tabular}{|l|l|}
118 \\
219,60
\end{tabular} & $\begin{array}{ll}129 \\
147,70 \\
\end{array}$ & \begin{tabular}{|l|l|}
145 \\
836,90
\end{tabular} & $\begin{array}{ll}150 \\
903,10 \\
\end{array}$ \\
\hline $\begin{array}{l}\text { 5. Средства образовательных организаций высшего образо- } \\
\text { вания Funds of educational institutions of higher education }\end{array}$ & 58,10 & 508,20 & 1568,80 & 891,80 & 1510,20 & 1824,90 & 2329,40 \\
\hline $\begin{array}{l}\text { 6. Средства частных некоммерческих организаций Private } \\
\text { non-profit organizations }\end{array}$ & 32,60 & 556,50 & 966,50 & 608,40 & 665,00 & 979,30 & 1269,10 \\
\hline $\begin{array}{l}\text { 7. Средства иностранных источников Funds from foreign } \\
\text { sources }\end{array}$ & 9172,40 & $\begin{array}{ll}18 \\
567,50\end{array}$ & $\begin{array}{ll}26 \\
145,50 \\
\end{array}$ & $\begin{array}{l}27 \\
750,70 \\
\end{array}$ & $\begin{array}{ll}22 \\
747,00 \\
\end{array}$ & $\begin{array}{ll}20 \\
982,40 \\
\end{array}$ & $\begin{array}{ll}24 \\
212,00 \\
\end{array}$ \\
\hline \multicolumn{8}{|l|}{ в том числе средства: including means: } \\
\hline $\begin{array}{l}\text { 7.1. Международных организаций } \\
\text { International organizations }\end{array}$ & $\cdots$ & 3682,10 & 4545,30 & 2455,70 & 1424,10 & 3104,60 & 1287,20 \\
\hline $\begin{array}{l}\text { 7.2. Государственных организаций зарубежных стран State } \\
\text { organizations of foreign countries }\end{array}$ & $\cdots$ & 5747,90 & 8 437,80 & 8494,40 & 4 582,90 & 4769,50 & 7221,90 \\
\hline $\begin{array}{l}\text { 7.3. Организаций предпринимательского сектора зарубеж- } \\
\text { ных стран The business organizations of foreign countries }\end{array}$ & $\cdots$ & 7893,30 & 8 107,40 & $\begin{array}{ll}11 \\
674,90 \\
\end{array}$ & $\begin{array}{ll}15 \\
525,50 \\
\end{array}$ & \begin{tabular}{|ll}
12 & 082, \\
10 & \\
\end{tabular} & \begin{tabular}{|l|l|}
14 \\
209,50 \\
\end{tabular} \\
\hline $\begin{array}{l}\text { 7.4. Прочих зарубежных организаций (организаций образо- } \\
\text { вания, фондов, некоммерческих организаций) Other foreign } \\
\text { organizations (educational institutions, funds, non-profit } \\
\text { organizations) }\end{array}$ & $\cdots$ & 1244,10 & 5 055, 10 & 5125,70 & 1214,40 & 1 026, 30 & 1493,50 \\
\hline
\end{tabular}

В процессе своей деятельности НПК может столкнуться с нехваткой собственного потенциала, необходимого для осуществления текущей операционной деятельности.

В этом случае:

1) рассматривается возможность привлечения к работам на временной основе соисполнителей, позволяя тем самым снизить загрузку собственных ресурсов, либо выполнить ряд работ, невозможных к осуществлению собственными силами НПК.

2) в рамках инвестиционной деятельности НПК оценивается необходимость расширения потенциала предприятия, посредством привлечения новых ресурсов.

Ограничениями по привлечению новых ресурсов в деятельность НПК являются:

- наличие возможности приобретения на рынке оборудования,

- состояние рынка занятости, отражающего возможность привлечения персонала;

- наличие средств как собственных, так и заемных, необходимых для приобретения и вовлечение в осуществляемый процесс вновь приобретенных ресурсов.
Важной особенностью при анализе возможности увеличения потенциала предприятия является учет характера используемых средств. Ими могут быть собственные средства, краткосрочные кредиты, долгосрочные кредиты и займы, средства, полученные от увеличения акционерного капитала, нераспределенная прибыль.

Использование каждого финансового ресурса налагает на предприятие свои условия и сроки возврата средств, которые учитываются при составлении бизнес-плана по увеличению на постоянной основе потенциала предприятия.

Процесс увеличения потенциала предприятия, как правило, носит постепенный характер, не предусматривающий скачкообразного изменения объемов осуществляемой деятельности. Это обстоятельство обусловлено прежде всего неопределённостью в долгосрочной перспективе спроса на продукцию конечным потребителем и макроэкономическим состоянием. Начальным этапом является необходимость учета состояния потенциала НПК и анализ возможностей по его увеличению. 


\section{Becmник BTYYMTI/Proceedings of VSVET, TIT. 79, № 2, 2017}

Следующим этапом изучается заказ на выполнение работ НПК, связанных с разработкой и производством инновационной продукции.

Заказ может поступать от различных государственных учреждений или иных предприятий и организаций, способных осуществить постановку технического задания и финансирование предстоящих работ, или заказ может быть собственной инициативой НПК, когда решение о разработке и внедрении инновационной продукции принимается по собственной инициативе предприятия и осуществляются на основании его способности произвести анализ в ожидаемой потребности товара конечным потребителем и оценкой структуры рынка.

Анализ типов заказов, реализуемых НПК, необходим при осуществлении планирования стратегии развития инновационной деятельности предприятия, которая прежде всего должна быть актуальна для конечного потребителя и отвечать процедурам привлечения возможности реализации проектов.

Участие подразделений НПК при выполнении инновационного заказа может быть различно.

Как правило, в рамках НПК формируются различные самостоятельные тематические направления, которые характеризуются определенным набором ресурсов, особенно кадровых. Поэтому каждый заказ анализируется с точки зрения соответствия его тематики определенному тематическому направлению предприятия. Однако, существуют заказы, требующие для своего выполнения ресурсов различных тематических направлений НПК. Работы по реализации подобных заказов являются исключительными для предприятия, а при повторении приводят к формированию нового тематического направления деятельности НПК.

Каждое направление деятельности имеет наибольшую стратегическую значимость, ввиду того, что техническая сложность полученной продукции высока, обеспечивая ее уникальность, больший уровень спроса и увеличивает время на выпуск товаров-аналогов конкурентами. Целесообразность принятия решения о разработке и последующем выпуске подобной продукции принимается при условии увеличения стоимости заказа по ее разработке в сравнении с текущими проектами, реализуемыми в этот момент НПК.

Уровень использования потенциала НПК определяется следующими факторами:

1. Способностью оценивать собственные возможности по выполнению работ и потребности конечного потребителя, позволяющей тем самым принимать заказы, соответствующие возможностям предприятия.

2. Эффективностью использования ресурсов предприятия при создании инновационной продукции: как собственных, так и соисполнителей, с целью распределения работ оптимальным образом.

Для проведения дальнейших рассуждений по установлению ключевых положений, лежащих в основе деятельности НПК, и выявления эффективных методов использования потенциала предприятия рассмотрим поэтапно алгоритм отбора заказов для включения их в план дальнейших работ (рисунок 1).

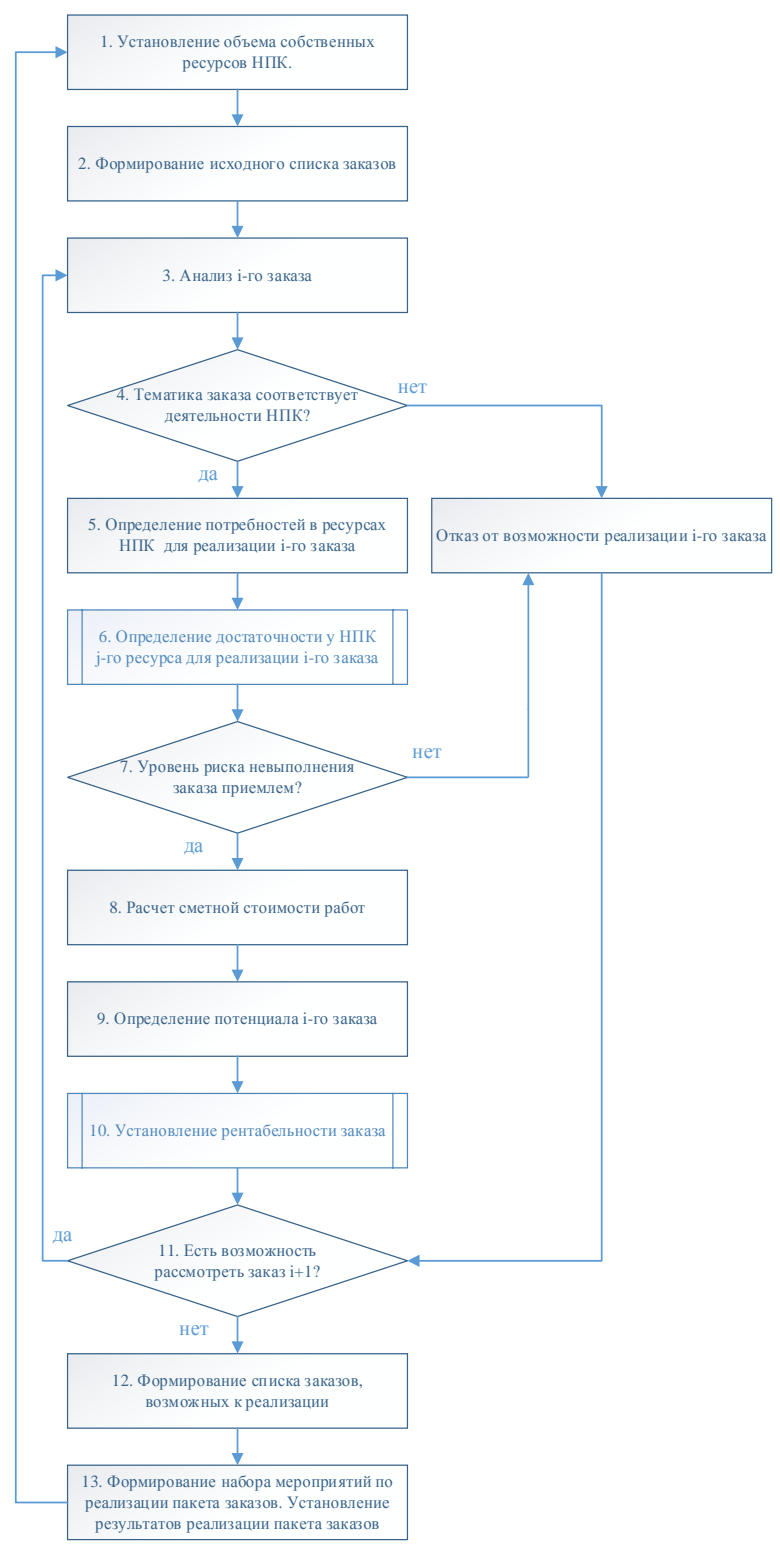

Рисунок 1. Алгоритм отбора заказов, реализуемых НПК

Figure 1. Algorithm of selection of the request realized by RPC 
Этап 1. Устанавливается набор ресурсов, которыми обладает НПК.

Так, в каждом тематическом направлении имеется определенный ресурс $R_{\mathrm{kj}}$, где $j-$ вид рассматриваемого ресурса $(j=1 \div n) ; k-$ тематическое направление $(k=1 \div m)$.

Рассматриваемый ресурс $R_{\mathrm{kj}}$ может быть представлен как:

- количество и тип имеющегося оборудования;

- число имеющегося персонала, осуществляющий определенный вид работ;

- прочее необходимое к рассмотрению.

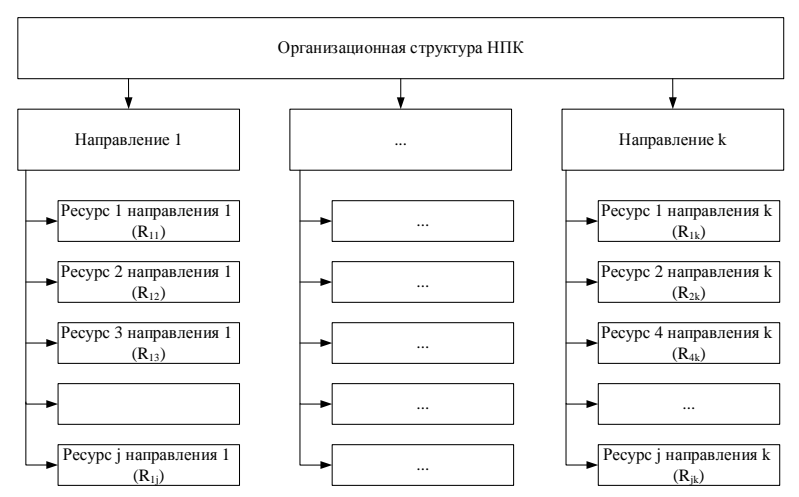

Рисунок 2. Схема учета собственных текущих ресурсов НПК

Figure 2. Scheme of the accounting of the RPC own current resources

Учет совокупного объема $j$-ого ресурса НПК $R_{\mathrm{j}}$ осуществляется:

$$
R_{j}=\sum_{k=1}^{m} R_{k j}
$$

Также определяется период $\mathrm{T}_{\mathrm{p}}$ в течение которого НПК необходимо спланировать свою деятельность, посредством подбора заказов, позволяющую предприятию реализовывать текущую конкурентоспособную деятельность. На практике, большинство НПК планируют свою деятельность на периоды в 1-3 года, ввиду специфики деятельности исследовательских организаций выраженной в специфике выполнении ОКР, а также неопределенностью таких факторов влияющих на спрос результатов деятельности предприятия, как поведение конкурентов, развитие научнотехнического прогресса, макроэкономические факторы и прочее.

Для установленного периода $T_{\mathrm{p}}$ определяется загрузка ресурсов согласно выполняемым предприятием работам. Тогда объем неиспользованных ресурсов для этого промежутка времени обозначим $R_{\mathrm{j}}\left(T_{\mathrm{p}}\right)$.
Этап 2. Формируется исходный список из $i$-ых заказов, возможных к реализации НПК, посредством сбора заявок на выполнение различных работ от иных предприятий, представителей государственных структур и прочие предложения, а также учитываются собственные проекты НПК.

Этап 3. Из сформированного в этапе 2 списка $i$-ых заказов каждый проект рассматривается поочередно, с целью выявления возможности и целесообразности его реализации.

Этап 4. Заказ проверяется на соответствие реализуемым тематикам научно-прикладной деятельности НПК. В случае несовпадения от возможности реализации заказа отказываются и рассматривается следующий проект.

Этап 5. Для заказа, тематика работ которого соответствует, устанавливается перечень требований к ресурсам НПК $\left(R_{\mathrm{jij}}\left(T_{\mathrm{p}}\right)\right)$, необходимых для его реализации.

Этап 6. Целью процедуры определения у НПК достаточности $j$-го ресурса, представленному на рисунке 3 , для реализации $i$-го заказа, является выявление возможности реализации работ по проекту.

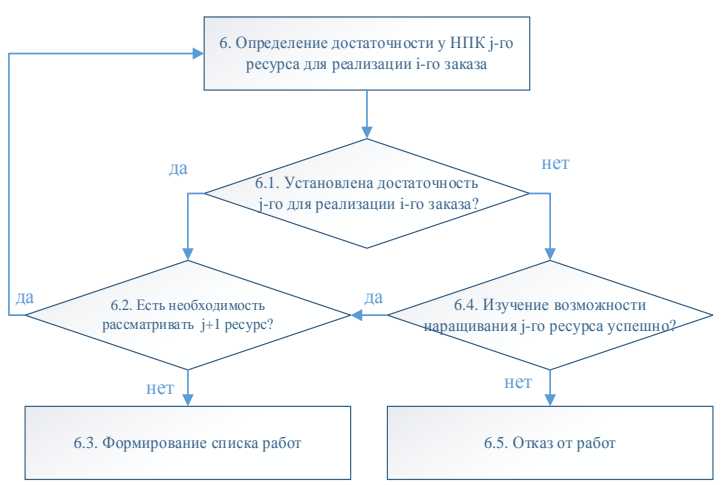

Рисунок 3. Алгоритм установления достаточности потенциала НПК для реализации $i$-го заказа

Figure 3. Algorithm of establishment of sufficiency of potential of RPC for realization of the bespoke

Этап 6.1. Сравнивается имеющееся требование к $j$-ому ресурсу для реализации $i$-го заказа с имеющимися возможностями.

$$
R_{\mathrm{ij}}\left(T_{\mathrm{p}}\right) \leq R_{\mathrm{j}}\left(T_{\mathrm{p}}\right)
$$

где $R_{\mathrm{ij}}\left(T_{\mathrm{p}}\right)$ - требование к $j$-ому ресурсу для реализации $i$-го заказа НПК; $R_{\mathrm{j}}\left(T_{\mathrm{p}}\right)-$ имеющиеся возможности по использованию $j$-го ресурса НПК.

Этап 6.2. В случае выполнения условия (2) определяется необходимость рассмотрения следующего ресурса $(j+1)$, требуемого для реализации $i$-го заказа.

Этап 6.3. После рассмотрения условия достаточности всех ресурсов, необходимых для реализации $i$-го заказа формируется список работ, необходимых к выполнению для реализации проекта. 
Этап 6.4. В случае несоответствия условия (2) устанавливается возможность наращивания потенциала НПК по $j$-му ресурсу посредством привлечения к работам смежников и соисполнителей, либо приобретения соответствующих материальных ресурсов.

Здесь может быть, как отдельная работа специалистов по проведению части исследовательских работ, так и выполнение части производственных операций по выпуску опытного образца или серийного производства. Работы смежников в этом случае рассматриваются как временная возможность по увеличению потенциала НПК для реализации $i$-го заказа.

Результатом анализа возможности привлечения смежников является установление возможности выполнения работ по $i$-ому заказу силами соисполнителей, которые не может реализовать НПК.

Этап 6.5. Если возможность увеличения объема необходимых ресурсов не установлена, то от реализации $i$-ого заказа отказываются.

Этап 7. Устанавливается риск невыполнения заказа, связанного с наличием различных факторов проведения работ по проекту:

- невыполнение работ подрядчиком по разработке технических и технологических решений, требуемых для выполнения заказа;

- срыв поставок полуфабрикатов и комплектующих требуемых для выполнения заказа;

- несвоевременная оплата заказчиком заказа;

- невозможность в отчетном периоде реализации работ, выполняемых по собственной инициативе;

- невыполнение проекта в целом, в котором участвует НПК как соисполнитель части работ;

- отсутствие положительных результатов при последовательном выполнении этапов работ по заказу, таких как НИР, ОКР;

- потеря необходимых сотрудников кадрового состава, необходимых для выполнения работ в отчетном периоде.

Влияние приведенных факторов на риск невыполнения заказа $\left(P_{\mathrm{i}}\right)$ определяется экспертной группой. Степень достоверности оценок экспертов проверяется установлением коэффициента конкордации [8].

Результаты оценки сравниваются с допустимым совокупным уровнем риска ( $\left.P_{\text {допуст }}\right)$ невыполнения подобных работ.

$$
P_{\mathrm{i}} \leq P_{\text {допуст }}
$$

В случае невыполнения условия (3) принимается решение об отказе реализации $i$-го заказа.

Этап 8. На основании сформированного списка работ рассчитывается сметная стоимость работ по выполнению $i$-го заказа, согласно порядку установленному в постановление правительства РФ от 17.02.2017 № 208.
Этап 9. Важным моментом при анализе деятельности НПК по выполнению заказов является учет фактора возникновения последующей заинтересованности у заказчика работ, в связи с вновь открываемыми перспективами применения полученных результатов от выполненных предприятием проектов. Эти перспективы выражаются в следующем:

1) увеличении жизненного цикла осуществляемых НПК работ за счет модернизации ранее выпущенной предприятием продукции;

2) возможности осуществлять работы определённого прикладного научно-тематического направления с меньшими сроками и затратами в сравнении с другими подобными НПК организациями.

Заказы, позволяющие получить подобные возможности для НПК можно отнести к разряду «перспективных».

Непосредственную оценку перспектив заказа необходимо производить с точки зрения оценки возможности дальнейшего использования результатов его реализации.

Если результаты могут быть достигнуты в следующем расчетном периоде деятельности НПК, то перспективы заказа целесообразно рассматривать как объем работ, возможный к реализации, выраженный в стоимостном выражении. Подобный подход обусловлен тем, что деятельность НПК планируется по дискретным периодам. Осуществление такого планирования базируется на оценке особенностей рынка, развития НТР, макроэкономической ситуации и т. п. Ввиду того, что перечисленные особенности разносторонние, предсказуемое планирование деятельности предприятия представляется целесообразным осуществлять на срок следующего планового периода.

Для случаев, когда результаты от выполнения заказа распространяются на более длительные промежутки времени, представляется возможным применить бальную экспертную оценку потенциала заказа. Результаты подобной бальной оценки проверяются расчетом коэффициента конкордации [8].

Этап 10. Данные о сметной стоимости работ и «перспектив» $i$-го заказа позволяют определить рентабельность его реализации, согласно алгоритму, приведенному на рисунке 4.

Установление величины прибыли заказа

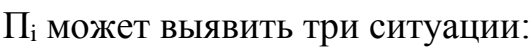

1) Значение прибыли имеет положительную величину $\Pi_{\mathrm{i}}>0$. В этом случае рассматриваемый $i$-ый заказ будет иметь положительную норму рентабельности и будет принят к дальнейшему рассмотрению возможности его реализации. 
2) Значение прибыли равно нулю $\Pi_{i}=0$. В этом случае выполнение заказа будет покрывать издержки на реализацию работ по проекту. Подобная ситуация соответствует условиям выполнения работ для точки безубыточности, позволяя также принять к дальнейшему рассмотрению возможность дальнейшего реализации $i$-го заказа. Как правило, возможность реализации такого заказа рассматривается если имеющиеся ресурсы загружены не полностью.

3) Значение прибыли имеет отрицательную величину $\Pi_{\mathrm{i}}<0$. Для этой ситуации рассматриваются мероприятия по проведению дополнительных переговоров с заказчиком по пересмотру цены, а также устанавливается «потенциал» проекта. Кроме того, эта ситуация будет распространяться на все собственные проекты НПК, ввиду того что реализация подобных работ осуществляется на средства предприятия.

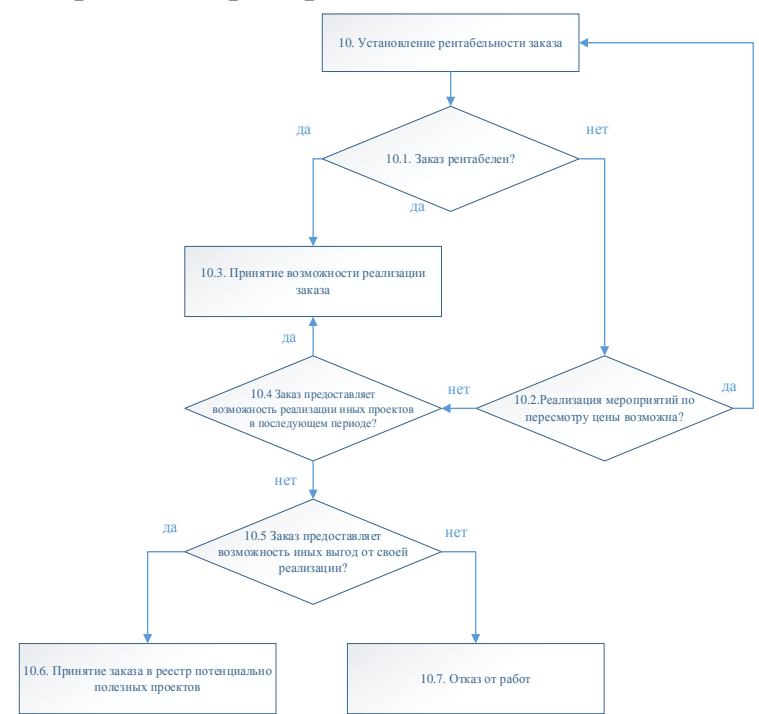

Рисунок 4. Алгоритм установления рентабельности i-го заказа

Figure 4. Algorithm of establishment of profitability of the bespoke

В случае отсутствия возможности пересмотра цены рассматривается «потенциал» $i$-го заказа, определенный в этапе 9. Для заказов с наличием «потенциала» принимается решение о возможности их дальнейшей реализации. Это обстоятельство обосновано тем, что НПК ведет прикладную научно-исследовательскую деятельность, а перспективные заказы в этом случае создают задел для подобных работ.

Решение о реализации «потенциально-полезных» заказов с отрицательным уровнем рентабельности зависит от текущих финансовых возможностей НПК и загрузки мощностей. При наличии таких возможностей первыми реализуются заказы, дающие результат в следующем расчетном периоде, как основного средства создания задела для инновационной деятельности предприятия. Затем рассматривается возможность выполнения заказов с более длительным периодом реализации.
Этап 11. После установления возможности реализации $i$-го заказа рассматривается следующий $i+1$ заказ из ранее установленного в п. 2 . списка работ.

Этап 12. Из прошедших отбор в возможности реализации НПК заказов формируется пакет проектов. При этом используется метод линейного программирования [1], так как основной задачей деятельности любого предприятия заключается в получении максимально возможного объема прибыли при ограниченных ресурсах.

Устанавливается функция $f(x)$ отражающая условия задачи по получению максимальной величины прибыли.

$$
f(x)=\sum_{i=1}^{p} \Pi_{i} \rightarrow \max
$$

где $i$ - рассматриваемый заказа $(i=1 \div p)$.

Условием ограничением в этом случае является наличие у НПК набора ресурсов, необходимых для реализации $i$-го заказа.

$$
\sum_{i=1}^{p} R_{i j}\left(T_{p}\right) \leq R_{j}\left(T_{p}\right)
$$

Тогда, условия транспортной задачи примут вид:

$$
\left\{\begin{array}{c}
f(x)=\sum_{i=1}^{p} \Pi_{i} \rightarrow \max \\
\sum_{i=1}^{p} R_{i j}\left(T_{p}\right) \leq R_{j}\left(T_{p}\right) \\
i \in I, I \cap p=\{1,2 \ldots, q\}
\end{array}\right.
$$

Произведенный отбор заказов позволит сформировать пакет проектов, возможный к реализации в период $T_{\mathrm{p}}$ с максимально возможным объемом прибыли.

Этап 13. Формируется набор мероприятий по реализации пакета заказов, включающий в себя набор операций по реализации отдельных видов работ, формированию методов мониторинга осуществляемой деятельности. Устанавливается влияние реализации пакета заказов на развитие потенциала НПК, с оценкой возможности изменения его потенциала и структуры.

\section{Заключение}

Рассмотренный алгоритм отбора заказов, позволяет установить следующие ключевые положения по организации деятельности НПК, влияющие на возможность использования его потенциала:

1. НПК- организация, осуществляющая научно-прикладную деятельность определенного тематического направления, результаты которой позволяют предприятию выпускать инновационную продукцию, с меньшими сроками разработки и внедрения в сравнении с иными организациями. Подобное свойство 
в «специализации» проведении работ определяет конкурентоспособность НПК и его жизнестойкость.

2. Имеющийся потенциал НПК позволяет ему реализовывать заказы с определенным уровнем требований к ресурсам предприятия, соответствующего уровня сложности и объемом производимых работ, выраженном в денежном эквиваленте. Изменение потенциала НПК влияет на возможности предприятия по реализации заказов.

3. В процессе своей деятельности НПК должен быть способен реализовывать «потенциальные» заказы, дающие результат в последующих периодах

\section{ЛИТЕРАТУРА}

1 Acemoglu D. Localised and Biased Technologies: Atkinson and Stiglitz's New View, Induced Innovations, and Directed Technological Change // The Economic Journal, T. 125, C. 443-463

2 John H. Reappraising the Eclectic Paradigm in an Age of Alliance Capitalism, The Eclectic Paradigm, C. 111-142

3 Pyka A., Foster J., The Evolution of Economic and Innovation Systems, Springer, 2015. C. 641.

4 Scott R. Baker Nicholas Bloom Steven J. Davis. Measuring Economic Policy Uncertainty // The Quarterly Journal of Economics, T. 131, C. 1593-1636

5 Kerr W., Lerner J., Stem S. Innovation Policy and the Economy / University of Chicago Press Joumals, 2015520 c.

6 Липина С.А., Липина А.В. Инновационная экономика 21 века: мировой опыт и практика // Успехи современной науки и образования. 2016. С. 11-13.

7 Мантатов К.Д. Тенденции развития предпринимательской сферы Российской Федерации под влиянием технологических инноваций // Финансовые проблемы обеспечения устойчивого роста современной российской экономики: Материалы Межвузовской научно-практической конференции Гатчина: Издво ЛОИЭФ, 2014. 19 с.

8 Мунасыпов Н.А. Линейное программирование, Оренбург: Агентство Пресса, 2015. 122 с.

9 Прохоров О.В. Проблемы внедрения технологических инноваций в коммерческих предприятиях Российской Федерации и возможные пути их решения // Международный научно-практический симпозиум молодых ученых и специалистов Экономическая политика современной России, СПб.: Изд-во ИМЦ НВШ-СПб, 2013. 289 с.

10 Российский статистический ежегодник. 2016: Стат.сб./Росстат. Р76 М., 2016, 725 с.

\section{REFERENCES}

1 Acemoglu D. Localised and Biased Technologies: Atkinson and Stiglitz's New View, Induced Innovations, and Directed Technological Change. The Economic Journal, vol. 125, pp. 443-463

\section{СВЕДЕНИЯ ОБ АВТОРАХ}

Елена А. Федорова д.э.н., директор, ООО «Универсал-Аудит», ул. Оружейная, 5а, г. Тула, 300012, Россия, eafokt@rambler.ru

Андрей Ю. Горбачев начальник сектора ценообразования, АО Центральное конструкторское бюро аппаратостроения, ул. Демонстрации, 36г. Тула, 300034,Россия, merc123@mail.ru

\section{КРИТЕРИЙ АВТОРСТВА}

Елена А. Федорова консультация в ходе исследования Андрей Ю. Горбачев написал рукопись, корректировал её до подачи в редакцию и несёт ответственность за плагиат

КОНФЛИКТ ИНТЕРЕСОВ

Авторы заявляют об отсутствии конфликта интересов.

ПОСТУПИЛА 08.04.2017

ПРИНЯТА В ПЕЧАТЬ 28.04.2017 в форме возможности обеспечения предприятием набором работ с определенным финансовым значением. Подобный задел по заказам образуется благодаря последовательно возникающему интересу у заказчика работ в результатах деятельности НПК и используется для обеспечения предприятия работой в последующих периодах.

Возможность НПК по реализации «потенциальных» заказов также влияет на его конкурентоспособность, отражая возможности предприятия по привлечению и реализацию заказов, стабильного спроса на результаты своей деятельности.

2 John H. Reappraising the Eclectic Paradigm in an Age of Alliance Capitalism, The Eclectic Paradigm, pp 111-142

3 Pyka A., Foster J. The Evolution of Economic and Innovation Systems, Springer, 2015, 641 p.

4 Scott R. Baker Nicholas Bloom Steven J. Davis. Measuring Economic Policy Uncertainty, The Quarterly Journal of Economics, vol. 131, pp. 1593-1636

5 Kerr W., Lemer J., Stem S. Innovation Policy and the Economy, University of Chicago Press Journals, 2015, 520p.

6 Lipina S.A., Lipina A.V. Innovative economy of the 21st century: international experience and practice. Uspekhi sovremennoi nauki i obrazovaniya [The success of modern science and education]. 2016. pp. 11-13. (in Russian).

7 Mantatov K.D. Tendentsii razvitiya predprinimatel'skoi sfery Rossiiskoi Federatsii pod vliyaniem tekhnologicheskikh innovatsii Finansovye problemy obespecheniya ustoichivogo rosta sovremennoi rossiiskoi ekonomiki: Materialy Mezhvuzovskoi nauchno-prakticheskoi konferentsii [Trends in the development of the entrepreneurial sphere of the Russian Federation under the influence of technological innovations the Financial problems of the sustainable growth of the Russian economy: materials of the Interuniversity scientific-practical conference]. Gatchina, LOIEF 2014. 19 p. (in Russian).

8 Munasypov N.A. Lineinoe programmirovanie [Linear programming]. Orenburg, Agentstvo Pressa, 2015. 122 p. (in Russian).

9 Prokhorov O.V. Problemy vnedreniya tekhnologicheskikh innovatsii v kommercheskikh predpriyatiyakh Rossiiskoi Federatsii i vozmozhnye puti ikh resheniya Mezhdunarodnyi nauchno-prakticheskii simpozium molodykh uchenykh i spetsialistov Ekonomicheskaya politika sovremennoi Rossii. St Petersburg, IMTs NVSh-SPb, 2013. 289 p. (in Russian).

10 Russian statistical Yearbook. 2016: Stat.sat./Russtat. 2016, 725 p. (in Russian).

\section{INFORMATION ABOUT AUTHORS}

Elena A. Fedorova doctor of economic sciences, CEO, "Universal-Audit”, Oruzheynaya str., 5a, Tula, 300012, Russia, eafokt@rambler.ru

Andrey Yu. Gorbachev chief of the sector of pricing, Central design office machine construction, Demonstration str., 36, Tula, 300034, Russia,merc123@mail.ru

\section{CONTRIBUTION}

Elena A. Fedorova consultation during the study

Andrey Yu. Gorbachev wrote the manuscript, correct it before filing in editing and is responsible for plagiarism

CONFLICT OF INTEREST

The authors declare no conflict of interest.

RECEIVED 4.8.2017

ACCEPTED 4.28.2017 